\title{
SYNDROME NEUROLOGIQUE MORTEL CHEZ UNE TOURTERELLE TURQUE (STREPTOPELIA DECAOCTO) ET FIXATION DE LA TIQUE (IXODES PARI (= I. FRONTALIS).
}

\author{
par C. CHASTEL*, J. Y. MONNAT**, G. LE LAY* et J. C. BEAUCOURNU***
}

\begin{abstract}
RÉSUMÉ. La paralysie à tiques chez les oiseaux sauvages est probablement fréquente mais rarement observée. Les auteurs rapportent le cas d'une tourterelle turque ( $S$. decaocto) chez laquelle se trouvaient associés un syndrome neurologique mortel et la fixation au-dessus de l'œil droit d'une femelle d'Ixodes pari. L'étude virologique des tissus de l'oiseau et de la tique a été négative. Il pouvait donc s'agir d'une paralysie à tiques ou d'un équivalent mineur de ce syndrome. Par ailleurs, c'est la première fois que la tourterelle turque est signalée comme hôte d'I. pari.
\end{abstract}

\section{Fatal neurological syndrome in a collared dove (Streptopelia decaocto) and fixation of Ixodes pari ( $=$ I. frontalis) tick.}

SUMMARY. Tick paralysis is probably a common condition in wild birds, but rarely observed. The authors report the case of a collared dove ( $S$. decaocto) presenting a fatal neurological syndrome associated with the fixation of a Ixodes pari female above the right eye. Virological studies of bird's tissues and of the tick were negative. These findings were compatible with tick paralysis or a minor equivalent of this syndrome. In addition, it was the first time that a collared dove is found as host for $I$. pari.

Le 3. 9. 1979, une tourterelle turque, Streptopelia decaocto, incapable de voler, est trouvée dans les jardins de la Faculté des Sciences de Brest. Elle présente un syndrome paralytique et une tique fixée au-dessus de l'œil droit.

La paupière supérieure droite est œdémateuse, obturant presque entièrement l'œil. La tique est une 우 d'Ixodes pari Leach 1815 (=I. frontalis), en oviposition. Elle est retirée à la pince, avec précautions, dans l'après-midi. Cedex.

* Laboratoire de Virologie, Faculté de Médecine, 22 avenue Camille Desmoulins, BP 815, F 29279 Bres,

** Laboratoire de Zoologie, Faculté des Sciences, avenue Victor Le Gorgeu, F 29283 Brest Cedex.

*** Laboratoire de Parasitologie, Faculté de Médecine, avenue du Professeur Léon Bernard, F 35043 Rennes Villejean Cedex.

Accepté le ro décembre r 980 . 
La tourterelle est placée dans une vaste cage avec des graines et de l'eau. Elle se tient debout, mais ne mange pas et ne boit pas. Elle exécute constamment des rotations sur elle-même, dans le sens inverse des aiguilles d'une montre.

Il existe une déviation permanente de la tête sur la droite et une déviation des rectrices, également à droite.

Le 4. 9. 1979, à 8 heures du matin, l'oiseau est trouvé mort dans sa cage. A l'autopsie, les poumons et le foie sont très congestifs. Des prélèvements sont faits en vue d'examens histopathologiques et virologiques.

\section{Histopathologie}

Au niveau de l'encéphale, il existe de la nécrose neuronale disséminée, sans réaction inflammatoire. Le foie et les poumons sont le siège d'une congestion massive. Les reins et le myocarde apparaissent normaux.

\section{Virologie}

Des suspensions de cerveau, de rein, de foie, de poumons et de myocarde sont inoculées par voie IC à des souriceaux ainsi qu'à des cultures de fibroblastes diploïdes humains MRC 5. La tique est également inoculée à des souriceaux.

Aucun virus n'a été isolé.

\section{Commentaires}

\section{I-Ornithologie.}

D'origine faunistique indo-africaine, $S$. decaocto connaît depuis le début du $20^{\mathrm{e}}$ siècle une importante phase d'expansion qui lui a permis d'envahir en quelques décennies la quasi-totalité de l'Europe où elle est maintenant couramment répandue dans les parcs et les jardins. Elle n'est pas exigeante pour son site de nidification : elle construit un nid sommaire fait de brindilles, le plus souvent sur des arbres ou des arbrisseaux, mais aussi parfois sur des poteaux télégraphiques ou des encadrements de fenêtre.

Elle niche désormais communément à Brest, notamment au-dessus du port de commerce où l'on peut voir depuis quelques années une impressionnante concentration d'immatures et de reproductrices ${ }^{5}$. L'origine de l'oiseau que nous avons étudié n'était donc probablement pas étrangère à la région brestoise.

\section{$2-$ Entomologie.}

Ixodes pari $(=I$. frontalis) est une tique spécifique des oiseaux et spécialement 
de ceux qui s'abritent, ou recherchent leur nourriture, dans les ronciers, les fourrés, etc.

Elle a été trouvée dans l'ouest de la France ${ }^{3}$ sur Phasianus colchicus, Rallus aquaticus et surtout sur des passereaux (Garrulus glandarius et des représentants du genre Turdus: $T$. viscivorus, $T$. philomelos, $T$. iliacus, $T$. merula, ainsi que sur Passer domesticus, ce dernier étant vraisemblablement un hôte accidentel, tout comme S. decaocto).

C'est en effet la première fois que l'on trouve $I$. pari fixée sur la tourterelle turque qui peut, par ailleurs, être parasitée par Ixodes ricinus ${ }^{2}$.

\section{3 - Pathologie.}

La paralysie à tiques est bien connue chez les mammifères : chien, mouton, homme. Elle semble souvent liée à la fixation d'une 우 en oviposition : I. ricimus, I. redikorzevi (=I. acuminatus), I. hexagonus, Dermacentor reticulatus, D. marginatus, dans nos régions.

Chez les oiseaux, elle est également connue, mais chez un oiseau dans la nature, elle a été rarement signalée sinon en Australie, après fixation d'Ixodes holocyclus (H. Hoogstraal, commun. écrite, 1980). On l'a observée surtout chez le poulet à la suite de la piqûre de divers Argas, du sous-genre Persicargas : A. (P.) walkerae, $A$. (P.) arboreus et $A$. (P.) radiatus ${ }^{4}$. Cette pathologie n'est pas ignorée du fait de ses conséquences économiques.

A notre connaissance, on n'a pas signalé de cas de paralysie à tiques chez des oiseaux, après piqûre de Ixodes pari ${ }^{1}$.

Bien que nous ne soyons pas absolument certains que les signes neurologiques que nous avons observés chez $S$. decaocto correspondent à une paralysie à tiques, la coexistence de ces troubles et de la fixation d'une + d'I. pari en oviposition évoque fortement ce syndrome, ou tout au moins un équivalent mineur de celui-ci. La tique a pu inoculer une neurotoxine responsable de la déviation de la tête et de la queue présentée par cet oiseau. Par contre, l'existence d'une atteinte encéphalitique, en particulier d'origine virale, paraît peu probable.

\section{BIBLIOGRAPHIE}

I. Arthur D. R. : British ticks, I vol., 2 I $_{3}$ p. Butterworths, Londres, 1963.

2. Ferianc O., Lichard M. : Birds in the Tribec and Hronsky Inove mountains as hosts of ticks.Bull. Wld Hlth Org., 1967, 36, suppl. 1, 19-23.

3. Grlot B., Beaucournu J.-C. : Premier inventaire des tiques d'oiseaux (Acarina, Ixodoïdea) del'ouest de la France; présence d'Ixodes unicavatus Neumann r9o8 en Bretagne. Bull. Soc. Sci. Bretagne, I973, 48, I3I-I4I.

4. Gothe R., Kunze K., Hoogstraal H. : The mecanisms of pathogenicity in the tick paralysies. J. Med. Entomol., I979, 16, 357-369.

5. Guermeur Y., Monnat J.-Y. : Histoire et géographie des oiseaux nicheurs de Bretagne. Ministère de l'Environnement et du Cadre de Vie, I vol., 240 p. Impr. Moderne, Aurillac, 1980. 